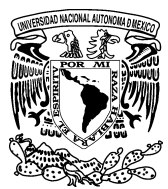

\title{
Diferencias en los motivadores y los valores en el trabajo de empleados en empresas maquiladoras
}

\author{
Differences in motivators and values in the work of \\ maquiladora industry employees
}

\section{Jorge Arturo Hernández Palomino*, José de Jesús Espinoza Medina y Manuel Aguilar Arellano}

\author{
Instituto Tecnológico de Ciudad Juárez, Ciudad de Juárez, México
}

Recibido el 12 de junio de 2014; aceptado el 5 de febrero de 2015

Disponible en Internet el 27 de octubre de 2015

\begin{abstract}
Resumen
Este estudio analiza con un diseño cuantitativo las diferencias en los motivadores y valores organizacionales de 4 generaciones de empleados que trabajan en la industria maquiladora en Ciudad Juárez. El estudio empírico se llevó en una muestra de 1,271 empleados en el año 2013. Para el análisis de la información y determinación de las posibles diferencias se utilizó la prueba Anova. Dado que la investigación se fundamentó en analizar las posibles diferencias en los motivadores entre las 4 generaciones de empleados en el estudio, se realizó un análisis factorial y rotación de Oblimin. Para la confirmación de dichas diferencias se utilizó la prueba $t$ con la corrección de Bonferroni. Los resultados proporcionan elementos diferentes que otros estudios han proporcionado. La existencia de diferencias significativas entre las 4 generaciones de empleados y una gran diferencia en la importancia que dan a los motivadores y valores organizacionales entre los empleados de las generaciones $\mathrm{X}$ e $\mathrm{Y}$ en comparación a los tradicionalistas y los baby boomers, representa información valiosa que académicos y empresas podrían considerar.

Derechos Reservados ( 2015 Universidad Nacional Autónoma de México, Facultad de Contaduría y Administración. Este es un artículo de acceso abierto distribuido bajo los términos de la Licencia Creative Commons CC BY-NC-ND 4.0.
\end{abstract}

Palabras clave: Recursos humanos; Valores generacionales; Motivadores

\footnotetext{
* Autor para correspondencia.

Correo electrónico: mt00831@yahoo.com.mx (J.A. Hernández Palomino).

La revisión por pares es responsabilidad de la Universidad Nacional Autónoma de México.
} 


\begin{abstract}
This study analyzes with a quantitative design the differences in the motivational and organizational values of four generations of employees working in the maquiladora industry in Ciudad Juarez. The empirical study used a sample of 1,271 employees in the 2013 year. For the information analysis and identification of possible differences the Anova test was used. Since this study is based on analyzing the differences in the motivators between four generations of employees of the study, a factorial analysis and Oblimin rotation was performed. To confirm these differences t test was used with Bonferroni correction. The results provide different elements that other studies have provided. The existence of significant differences between the four generations of employees in the importance given to the motivational and organizational values represents valuable information that academics and companies could consider.

All Rights Reserved () 2015 Universidad Nacional Autónoma de México, Facultad de Contaduría y Administración. This is an open access item distributed under the Creative Commons CC License BY-NC-ND 4.0.
\end{abstract}

Keywords: Human resources; Generation values; Motivators

\title{
Introducción
}

La motivación permanece como uno de los mayores problemas que enfrentan las organizaciones de hoy, considerando que los empleados motivados son esenciales para el éxito de una organización (Robbins, 2004). Investigaciones realizadas muestran que los trabajadores altamente calificados que se sienten satisfechos con sus actuales trabajos representan un $25 \%$ más de aquellos trabajadores que no lo están (Cialdini, Petrova y Goldstein, 2004). Dado que la motivación es un elemento clave de la satisfacción en el trabajo, de la productividad y del desempeño, existe una larga lista de estudios académicos y teorías, por ejemplo las de Hackman y Oldham (1980), Herzberg (1959), McClelland (1961), Maslow (1963) y Vroom (1964).

A pesar del progreso que se ha realizado en identificar los factores que influyen en la motivación en el trabajo, poner este conocimiento en la práctica podría convertirse en una tarea retadora al aplicarlo en una fuerza de trabajo multigeneracional (Latham y Pinder, 2005). A principios de la década del 2000, los profesionistas más jóvenes que nacieron después de 1980 se empezaron a contratar en las organizaciones, convirtiéndose en la cuarta generación de trabajadores calificados que trabajan juntos en las empresas. Estas 4 generaciones de los tradicionalistas (nacidos antes de 1946), los baby boomers (nacidos entre 1946 y 1964), la generación X (nacidos entre 1965 y 1979) y la generación Y (nacidos después de 1979) han empezado a coincidir en las organizaciones con sus propias necesidades, valores y creencias (Eisner, 2005).

Adicionalmente a estas diferencias entre generaciones, los gerentes de las empresas están enfrentando el reto del ingreso de una amplia fuerza calificada de la nueva generación X y un flujo muy amplio de la fuerza laboral de los baby boomers que se acerca a sus años de retiro. Considerando este aspecto demográfico, los dirigentes de las empresas continúan en la búsqueda de maneras de mitigar los efectos del éxodo masivo de los baby boomers y los tradicionalistas.

Se ha predicho que los efectos incluyen cambios significativos en la diversidad generacional de la fuerza de trabajo, a medida que la generación X comience a reemplazar de forma más significativa los empleados de la generación de los baby boomers (O’Bannon, 2001), así como los diferentes valores generacionales que se presentan en la fuerza laboral de las empresas hoy en día. 
El punto es que la combinación de un gran grupo de jóvenes trabajadores entrando y un gran grupo de trabajadores experimentados que están dejando las empresas forzará a las empresas a encontrar mejores estrategias para continuar utilizando la experiencia y el conocimiento de los baby boomers que empiezan a retirarse, retener a los mejores talentos y experiencia de la generación X y fortalecer a los nuevos integrantes sin experiencia, representados por la generación Y.

La industria maquiladora, una de las muchas industrias que están siendo impactadas por este fenómeno demográfico, se encuentra altamente preocupada por el problema potencial de perder a empleados valiosos en los próximos 10 años. Los gerentes de las empresas, los gerentes de recursos humanos y los gerentes de operaciones en la industria están enfrentando el dilema de cómo reemplazar a esta fuerza de trabajo en retiro que representan los baby boomers. Del otro lado encontramos la continuidad generacional, la generación Y, que ha empezado a graduarse de sus estudios universitarios y podría llenar los espacios dejados.

Por lo tanto, capacitar y desarrollar a un grupo de nuevos graduados para llenar las vacantes dejadas por el éxodo de un grupo experimentado de baby boomers en la industria se presenta como un reto. Con las crisis experimentadas por la industria maquiladora en los años 2000 y 2007, la fuerza laboral de dicha industria en Ciudad Juárez perdió 92,000 empleos. Debido a ello, la baja tasa de contratación de profesionistas y el creciente despido de otros muy jóvenes y con escasa experiencia los forzó a tratar de insertarse en otras industrias. Hoy, en 2014, esos empleos se han recuperado y se tiene un máximo histórico de 252,809 empleados.

Considerando estos retos, un estudio para investigar y comparar los motivadores en el trabajo y valores organizacionales de las diferentes generaciones de profesionistas que trabajan en la industria maquiladora es oportuno. Resulta fundamental determinar la diferencia existente entre los motivadores en el trabajo y los valores organizacionales relativos a la satisfacción en el trabajo, la productividad y el desempeño respecto a los factores de decisión que los trabajadores consideran tanto para contratarse con una empresa, como para decidir retirarse de ella (Sutton y Griffin, 2004).

El aspecto a considerar en la motivación en empleados de mayor edad se convierte más relevante a medida que la generación conocida como baby boomers se empiece a retirar. Los baby boomers son 2 generaciones: una de 1946 a 1953 y la otra de 1954 a 1964. Nacieron en el periodo de posguerra y en general han disfrutado de una prosperidad económica a lo largo de su vida productiva. A este grupo se le considera uno de los que más han avanzado, y su legado tecnológico y económico es el mayor registrado en la historia.

Esto provocará lagunas en el conocimiento, así como escasez de mano de obra especializada que influye en la toma de decisiones en las organizaciones. Los más viejos baby boomers cumplieron 65 años al finalizar el año 2011. Los miembros más jóvenes de dicha generación tendrán una edad de 65 en el año 2029. Esta población, de aproximadamente 21 millones de personas, es mayor al corte de la generación siguiente, conocida como generación X.

Entender estas diferencias puede ayudar a los responsables de las empresas a crear entornos propicios para la satisfacción laboral de 4 generaciones que confluyen diariamente en sus actividades laborales. Adicionalmente, cuando las políticas y los valores de la empresa son comunicados adecuadamente pueden jugar un rol clave en el éxito de una estrategia de personal diseñada para ser dirigida a ciertos segmentos particulares, tales como los ingenieros y los administradores de las empresas.

El trabajo se encuentra organizado como sigue: la revisión de la literatura aborda el marco teórico y los estudios empíricos previos, y a partir de dicha revisión se plantean las hipótesis; posteriormente, se presenta la metodología utilizada; enseguida se presentan los resultados de 
la investigación; finalmente, se muestran las conclusiones, limitaciones y recomendaciones para futuras investigaciones.

\section{Revisión de la literatura}

La motivación ha disfrutado de un largo y completo interés de académicos y empresarios, lo cual ha redituado en una abundante cantidad de teorías. Entre las diferentes teorías, 5 han destacado por los elementos que presentan. Maslow (1943) presentó la teoría psicológica de las necesidades humanas en las que establece una jerarquía de necesidades humanas y defiende que, conforme se satisfacen las necesidades más básicas (parte inferior de la pirámide), los seres humanos desarrollan necesidades y deseos más elevados (parte superior de la pirámide). McGregor (1960) introdujo la teoría X e Y, en la que distinguió 2 alternativas sobre las personas. Por un lado, que los seres humanos son perezosos y, por lo tanto, deberían ser motivados a través del castigo; a esto lo consideró la teoría X. Por el otro lado, la teoría Y suponía al ser humano con una naturaleza al compromiso y a la búsqueda de responsabilidades. Vroom (1964) introdujo la teoría de las expectativas, en la que consideraba que los individuos como seres pensantes tienen creencias y expectativas respecto a los sucesos futuros de sus vidas.

Adicionalmente, Herzberg (1968), en su teoría de los 2 factores sostiene que por una parte hay factores higiénicos o factores extrínsecos, que están relacionados con la insatisfacción, pues se localizan en el ambiente que rodean a las personas y abarcan las condiciones en que desempeñan su trabajo, y por otra parte hay factores motivacionales o factores intrínsecos, relacionados con la satisfacción en el cargo y con la naturaleza de las tareas que el individuo ejecuta.

McClelland (1961) propuso una mirada diferente a lo que antes había como teorías de las motivaciones sociales. Según McClelland, el individuo adopta experiencias, las cuales se traducen en nuevas formas de entender el medio, lo que se llama comúnmente teoría de las necesidades secundarias. Dichas necesidades estaban fundadas en el logro, en la afiliación y en el poder.

Desde el punto de vista psicológico, las motivaciones son el conjunto de factores que impulsan el comportamiento de los seres humanos hacia la consecución de un objeto. Por ejemplo, una persona tiene muchas necesidades en cualquier momento. Algunas son el resultado de estados fisiológicos de tensión como el hambre, la sed y la incomodidad. Otras, resultado de los estados psicológicos de tensión como la necesidad de reconocimiento, estimación o pertenencia.

Las aportaciones de la psicología y de otras ciencias sociales han puesto de manifiesto la relevancia del factor humano, de las relaciones humanas, y también la necesidad de tomar en consideración la realidad sociotécnica del sistema de trabajo y el valor de una gestión de los recursos humanos inspirada en los principios humanistas (Peiró, 2004).

Empleados motivados que están satisfechos con sus trabajos son esenciales para el éxito organizacional. Estudios anteriores, como el realizado por Cialdini et al. (2004), han mostrado que empleados altamente calificados que están satisfechos en su trabajo llegan a mejorar su desempeño en un $25 \%$ más que los que no están motivados. De la misma manera, demuestran que los valores de los más satisfechos son diferentes a los que no están motivados.

Los valores son un aspecto profundo del ser humano (Rokeach, 1973) y continuarán siendo un aspecto importante en la investigación de la administración de las empresas, porque son fundamentales para entender la motivación de los empleados y la satisfacción en el trabajo (Karl y Sutton, 1998). A pesar de que se han realizado progresos importantes en predecir, entender y determinar la influencia de la motivación en el lugar de trabajo, mantener a la gente motivada es uno de los mayores problemas que enfrentan las organizaciones en la actualidad. 
A pesar de todas estas grandes teorías en las que nos hemos apoyado por largos años, parece que no nos sirven totalmente en este momento. Lo que hoy se hace necesario es abordar los fenómenos como lo plantea Santos (2006). El autor sostiene que lo que se requiere hoy en día es una ecología de saberes. Es muy importante entonces hacer este cambio, de una epistemología basada solamente en una forma de conocimiento a otra de ecología. Cuando hay una ecología de saberes, la ignorancia no es necesariamente un punto de partida, puede ser un punto de llegada.

El primer reto es reinventar las posibilidades emancipatorias que estaban en este conocimiento emancipador: una utopía crítica. Construir la emancipación a partir de una nueva relación entre el respeto de la igualdad y el principio del reconocimiento de la diferencia (Santos, 2006). Toda la energía emancipatoria teórica fue orientada por el principio de la igualdad, no por el principio del reconocimiento de las diferencias. Ahora tenemos que intentar una construcción teórica donde las 2 estén presentes, y saber que una lucha por la igualdad tiene que ser también una lucha por el reconocimiento de la diferencia, porque lo importante no es la homogeneización sino las diferencias iguales.

Las transformaciones sociales, económicas y tecnológicas suponen un nuevo contexto de la actividad empresarial y productiva. La globalización de la economía, la internacionalización de los mercados, la diversificación de las demandas y preferencias de los consumidores y la fuerte competencia plantean exigencias cada vez mayores a las empresas en su productividad, calidad, reducción de costos, innovación, distribución, comercialización y otros aspectos relevantes. Estas exigencias requieren a su vez actuaciones de las empresas en diversos ámbitos y, muy particularmente, en las formas de organizar el trabajo.

Además, los cambios tecnológicos, cada vez más fuertes y frecuentes, también tienen importantes consecuencias para la reorganización del trabajo y las actividades que lo configuran. Todos estos cambios requieren una gran capacidad de adaptación de los trabajadores y una gestión mucho más flexible de los recursos humanos por parte de la empresa.

Actualmente, las empresas deben buscar la mejor manera de administrar una fuerza laboral multigeneracional con distintos valores, creando un desafío en la comunicación y el trabajo en equipo. Las empresas deben considerar los factores psicosociales que pueden adoptar valores por exceso, por defecto o por configuración adversos o desfavorables que pueden afectar negativamente a la salud y seguridad de los trabajadores, así como al desarrollo del trabajo, entendiendo por factores psicosociales aquellas condiciones que se encuentran presentes en una situación laboral y que están directamente relacionadas con la organización, el contenido del trabajo y la realización de la tarea (Juárez, 2007).

Establece Kyles (2005) que cuando múltiples generaciones trabajan juntas en un departamento o un equipo, pero que no entienden o no conocen los valores generacionales de sus colegas, la producción explosiona y salen chispas. Las diferencias generacionales han existido a lo largo del tiempo; sin embargo, el estudio de la historia desde la perspectiva de diferentes generaciones no tenía mucho sentido hasta hace relativamente poco tiempo, debido a la lentitud de la vida y los cambios casi imperceptibles entre una generación y la siguiente (Schlesinger, 1986).

Las diferencias entre generaciones han llegado a ser más pronunciadas con el surgimiento de la revolución industrial, que nos llevó a la era de la información y al ritmo vertiginoso en que vivimos hoy en día. El primer teórico en desarrollar sistemáticamente, a principios del siglo xx, el tema de las generaciones como parte de la consciencia histórica y la organización social fue Mannheim (Bengtson, Furlong y Laufer, 1983). Señalan los mismos autores que fue Mannheim (2013) quien, a través del estudio sociológico de las generaciones, proporcionó un medio para expresar que el ritmo del desarrollo histórico tiene sus bases en la ley biológica de la vida útil limitada del hombre y la superposición de las nuevas y viejas generaciones. 
En medio de esta concurrencia de 4 generaciones en los lugares de trabajo, Lipovetsky (1990) concluye que vivimos en una era del vacío en la que la sociedad posmoderna individualista sustentada en valores materiales no encuentra un fin o metas colectivas, solo un fin individual, lo que no le proporciona ningún tipo de satisfacción. Para el autor, el Superyó se presenta actualmente bajo la forma de imperativos de celebridad, de éxito, que de no realizarse desencadenan una crítica implacable contra el Yo (Lipovetsky, 1998). De este modo se explica la fascinación ejercida por los individuos célebres e ídolos, estimulada por los medios de comunicación, que intensifican los sueños narcisistas de celebridad y de gloria, animan al hombre de la calle a identificarse con las estrellas, a odiar el borreguismo y le hacen aceptar cada vez con más dificultad la banalidad de la existencia cotidiana.

Hoy en día, las organizaciones tienen que enfrentar lo que Bauman (1999) llama la sociedad líquida. El autor plantea como el ser humano ha transformado la esencia del trabajo como bien común a la esencia del trabajo individual. El trabajador pasa de un trabajo duradero sin vínculos y agradecimiento, a un trabajo inmediato en que los vínculos desaparecen y la gratitud instantánea es lo único que considera.

Lo anterior tiene fundamento en el punto de vista psicosocial, tal como lo plantea Peiró (2004) al abordar los cambios recientes en el entorno económico y social. Establece el autor que las transformaciones sociales, económicas y tecnológicas han generado un nuevo panorama en la actividad de las empresas. Dentro de este panorama se pueden identificar los movimientos de grupos de personas, donde el cambio de territorio tiene sus propias motivaciones, modalidades, consecuencias, significaciones y sueños (Bustillos, Rincones, Jiménez, Hernández, 2009).

Lo movimientos migratorios actuales tienen un perfil totalmente diferente al de otros tiempos, por lo que es posible aventurar la idea de una nueva época de las migraciones, por su relevancia y sus implicaciones a nivel no solo local, sino regional y nacional. Durand (2005) postula que México es un país de migrantes que históricamente no se reconoce como tal. Al igual que las manifestaciones particulares que ha asumido la migración México-Estados Unidos, las percepciones sociales e institucionales al respecto también han experimentado variaciones a lo largo de la historia.

En México, la salida de trabajadores calificados de diferentes generaciones al extranjero se mueve entre la retórica oficial de falta de reconocimiento y su aplastante realidad (Bustillos et al., 2009). La razón de esta salida de trabajadores está relacionada con la búsqueda de mejores empleos con mayores niveles de remuneración, un mayor reconocimiento al trabajo realizado y un medio ambiente más seguro y estable (Mckenzie y Sasin, 2007).

En consistencia con Santos (2006), de que las creencias o teorías no son un reflejo del mundo actual, la empresa maquiladora de Ciudad Juárez se ha vuelto un semillero de gente talentosa y bien pagada que abarca 4 generaciones de empleados en el mismo lugar de trabajo y que en lo general no aspira al famoso sueño americano (Bustillos et al., 2009). Si en alguna industria trabaja una gran parte de trabajadores extranjeros, esa es la industria maquiladora asentada en Ciudad Juárez. Hoy existen 322 empresas maquiladoras que a julio de 2014 emplea a 252,809 empleados (AMAC, 2014).

Dividir a la gente en generaciones provoca la objeción obvia desde que los niños nacen y mueren en una línea de tiempo continua, considerando que la categorización de las generaciones es arbitraria (Schlesinger, 1986). Con los años abundan otros problemas; por ejemplo, cada generación es conocida por varios nombres y tiene un inicio divergente que inicia y termina dependiendo del investigador o autor (Markert, 2004).

La confusión persiste, sin embargo, en la obtención de un consenso para la definición de la cohorte generacional. Mannheim (2013) recomienda un período de 30 años para cada generación, un período de tiempo suficiente para que cada generación pueda madurar y aprender lo suficiente 
para ser influyente. La teoría contemporánea, sin embargo, sostiene que una generación es más o menos cada 20 años, pero todavía hay grandes divergencias en las definiciones.

La fuerza de trabajo de hoy, por primera vez en la historia, se compone de 4 generaciones (Eisner, 2005), y presenta nuevos retos en las estrategias de organización para atraer, motivar y retener a los empleados (Earle, 2003; Harris, 2005). Hacer frente a estos retos es esencial para mantener la armonía, la productividad y la estabilidad dentro de las organizaciones. El problema, por supuesto, se debe a las diferencias de los valores que dan lugar a las diferentes expectativas de los lugares de trabajo, uno mismo, y los compañeros de trabajo.

Aunque las fechas y nombres de las generaciones difieren para la misma generación, las descripciones de las generaciones son muy consistentes (Eisner, 2005). Las diferencias en la asignación de rangos de edades o fechas parecen surgir de 2 aspectos principales. Uno de ellos es la confusión entre los términos de cohortes y generación, que se utilizan frecuentemente en la literatura de forma indistinta (Markert, 2004). El segundo es el sistema de asignación de grupos generacionales sobre la base de compartir experiencias tempranas sociales y económicas.

Los tradicionalistas nacidos antes de 1946 son el más pequeño y más viejo segmento de la fuerza de trabajo y comprenden aproximadamente el $4 \%$ de la población laboral. Los tradicionalistas son conocidos también como «la gran generación», «la generación olvidada», «la generación de la segunda guerra mundial». Algunos de los eventos más característicos que dieron forma a cada una de las generaciones es que a los tradicionalistas les tocó vivir la Segunda Guerra Mundial, la Gran Depresión, escuchaban más la radio y la televisión, anteponen el trabajo al placer, se sacrifican por la empresa sin muchas peticiones, y probablemente han tenido el mismo trabajo toda la vida.

La mayoría de los tradicionalistas se encuentran sobre los 65 años de edad y se encuentran en la fase más rápida de retiro entre las 4 generaciones (Morton, 2004). Aunque son la fuerza de trabajo que se está retirando en mayor número, son los más ricos, son los mayores contribuyentes e incluso son profesores de alto nivel en muchas universidades. En general los tradicionalistas son capaces de resolver problemas, se caracterizan por un buen sentido del humor, poseen fuertes valores patrióticos y familiares, y permanecen en un trabajo por largo tiempo (Morton, 2004).

Como empleados, los tradicionalistas están satisfechos con el trabajo bien hecho, prefieren líneas claras de autoridad y, a pesar de que muestran una fuerte independencia, cuando los ponen a cargo hacen lo correcto en caso de duda (Harris, 2005). Los tradicionalistas tienen una fuerte ética de trabajo, y son frugales, resultado de haber crecido en tiempos de escasez económica y turbulencia política (Eisner, 2005). Aunque la mayoría se ha retirado, dejan en el lugar de trabajo grandes logros. Sobrevivieron a la gran depresión económica, la Segunda Guerra Mundial y armaron una paz duradera. Ellos apoyaron a construir una infraestructura nacional y han contribuido con grandes inventos médicos y vacunas.

La generación de los baby boomers, nacidos entre 1946 y 1964, comprende entre el 40 y el $48 \%$ de la fuerza de trabajo y son el grupo más grande en la fuerza laboral de hoy (Eisner, 2005). A pesar de que ellos representan un gran número potencial de jubilaciones en los próximos 10 a 15 años, no significa necesariamente que los baby boomers se jubilarán por completo. De acuerdo con Reynolds (2004), el $31 \%$ de los baby boomers se están moviendo a nuevas líneas de trabajo y el $10 \%$ de ellos han empezado a trabajar en servicios personales como consultores, asesores financieros y de negocios.

Este tipo de trabajos permite una mayor autogobernancia, a la cual apelan los baby boomers por mantener la independencia (Reynolds, 2004). Como generación, comparten la experiencia del surgimiento de la televisión a color, el movimiento de los Derechos Civiles, la liberación de la mujer, el movimiento estudiantil del 68, la prosperidad económica, el asesinato de John 
F. Kennedy, el fenómeno de los Beatles, disturbios políticos, la llegada del hombre a la Luna, la guerra de Vietnam, protestas contra la guerra, la experimentación social, la libertad sexual, el movimiento ecologista, el movimiento feminista, protestas y disturbios, la experimentación con diversas sustancias tóxicas recreativas.

Estos eventos dieron forma a crear una generación caracterizada por trabajar duro, a la que le gustan los títulos; son independientes, optimistas, políticamente activos y con una actitud de poder hacer. Los baby boomers muestran lealtad, trabajan muchas horas, son altamente competitivos y miden el éxito de forma material. A pesar de ser altamente respetuosos de la autoridad, son propensos a resentir el autoritarismo o la pereza y tienden realizar sus gestiones basados en el tiempo (Morton, 2001). Los baby boomers son buenos en las relaciones y trabajando en equipo, no son buenos en los presupuestos y no les gusta el conflicto. Suelen anteponer el proceso a los resultados y pueden ser críticos y egocéntricos (Zemke, 2001). Suelen medir el éxito del trabajo por títulos en el trabajo, promociones, oficina y actividades que fomentan la autorrealización. Han cambiado dramáticamente y popularizado cada etapa de sus vidas, negándose a respetar la tradicional edad para retirarse.

La generación X, los nacidos entre 1965 y 1980, representan el 34\% de la fuerza de trabajo. A diferencia de otras generaciones de la fuerza de trabajo hoy en día, la generación $\mathrm{X}$ ha recibido una caracterización un tanto negativa, tanto en los medios de comunicación como en la literatura (Morton, 2003). Descripciones tales como perezosos, cínicos y quejosos (Williams y Nussbaum, 2001) se han incluido con descriptores más positivos, tales como inteligentes, independientes, pragmáticos y autosuficientes (Morton, 2003); individualistas, enérgicos, ambiciosos emprendedores, amantes de la libertad y autosuficientes (Eisner, 2005).

La generación X crece ya en un mundo en línea las 24 horas, los 7 días a la semana, con el teléfono celular, el cable e Internet y viviendo en hogares con altas tasas de divorcios. Ellos no tienen héroes. Todos los referentes de la generación son buenos y malos al mismo tiempo: Bill Clinton, Madonna, Michael Jackson, Bill Gates, Maradona, los Simpsons. . ¿En qué creen? En ellos mismos. Son los «Just do it» de Nike. Es una época de ruptura de grandes paradigmas: la caída del Muro de Berlín, la explosión del Challenger, los ataques terroristas en las Olimpíadas de Múnich, el suicidio en masa de Jonestown, el asesinato de John Lennon, los despidos masivos de los ochenta y las crisis económicas. Se crían en un mundo violento y conviven con las drogas y el sida.

Cuando ellos eran niños, fueron criados por la televisión mientras sus padres trabajaban. Un tercio de la generación X creció en la pobreza causada por la crisis económica, el desempleo y las altas tasas de divorcio (Morton, 2003). La generación X se crió en una época en que las necesidades de los niños fueron puestas al final y sobrevivieron la década de 1970 con agitaciones económicas. El aspecto positivo de la generación X es que crecieron en un mundo relativamente pacífico, en el auge tecnológico y disfrutando de los beneficios de las causas que sus padres iniciaron, como la de los Derechos Civiles, los movimientos de las mujeres y la protección al medio ambiente (Eisner, 2005).

Esta generación piensa globalmente, está tecnológicamente preparada, es divertida, informal y pragmática (Zemke, 2001). Ellos tienden a mantener un equilibrio entre vida y trabajo. Se les reconoce por la frase: «Trabajar para vivir, no vivir para trabajar» (Sturges y Guest, 2004). Son individualistas y ambiciosos, y en 1990 su espíritu emprendedor los convirtió en los superestrellas del punto-com. Los miembros de la generación X miden el éxito a través de trabajos flexibles que les permitan equilibrar el trabajo y la vida, para mantener relaciones sanas y estables (Morton, 2003). 
La generación Y, nacida entre 1981 y 2000, representa el 20\% de la fuerza laboral, y por sí sola será la generación que llene el vacío que vayan dejando los baby boomers que se vayan retirando. Si la generación X puede ser considerada como la generación perdida, viviendo a la sombra de los baby boomers, la generación Y puede ser considerada la generación encontrada, quienes fueron adorados por sus padres de la generación $\mathrm{X}$ y altamente apreciados por sus abuelos de la generación de los baby boomers (Zemke, 2001).

La generación Y presenció los ataques a las torres gemelas en Estados Unidos, conocido como el 9/11. Esta generación se distingue por una actitud desafiante y retadora. Esta fue la última generación que vio la Guerra fría por los pocos recuerdos que tiene desde la niñez, en tecnología le tocó vivir el auge de Internet, la transición del DOS al Windows, jugó desde el Atari hasta el wifi.

Los miembros de la generación Y crecieron siendo el centro de atención de familias ocupadas con vidas sobreplaneadas. En un mundo comunicado las 24 horas, los 7 días de la semana, con tecnología digital, la generación $\mathrm{Y}$ ha visto más a una edad menor que cualquiera de otra generación (Sujansky, 2004). Son usuarios consumados de la tecnología y crecieron con Internet. Acostumbrados a estar conectados con amigos y familiares en todo momento, los miembros de la generación Y son hijos de padres muy involucrados que los valoran y han reducido la brecha generacional entre ellos.

Los valores y las actitudes de la generación Y son la lealtad, la inclusión y la autosuficiencia (Eisner, 2005). Es la generación más conocedora de la tecnología, y como tales son más abiertos al trabajo en equipo que cualquiera de las generaciones anteriores y se desarrollan en la creación de redes e intercambio de información. La generación Y mide el éxito a través de la realización personal en el trabajo, una vida activa fuera del trabajo y su participación en la comunidad. Es la generación mejor educada, y si se le agrega su sofisticación tecnológica, las expectativas positivas y su aparente inclinación colectiva, es probable que tengan la fórmula para la grandeza (Zemke, 2001).

Para administrar de manera efectiva en este entorno, los gerentes de recursos humanos deben crear políticas flexibles y procedimientos que se adapten a todas las generaciones, algo que no ha sido el estándar para las organizaciones en el pasado. La mayoría de las organizaciones todavía están luchando para realizar los cambios necesarios y competir en la era del conocimiento (Ware y Grantham, 2003), y esta lucha se extiende a los cambios necesarios en la administración de recursos humanos. Los gerentes de las empresas tienen una verdadera oportunidad de sobresalir ejecutando los cambios necesarios para motivar y retener a los empleados en un ambiente de trabajo multigeneracional.

Las nuevas formas de organizar el trabajo buscan una mayor eficiencia y eficacia, una mayor flexibilidad, una optimización de los recursos tecnológicos y una mayor productividad y calidad como diferencia competitiva de la empresa. La gestión de la calidad total, la exigencia de cero defectos, el justo a tiempo, la reingeniería de procesos, el adelgazamiento de las organizaciones, los grupos semiautónomos de trabajo, los equipos de trabajo autodirigidos, el trabajo telemático, la externalización de la producción y la gestión flexible de esa producción son estrategias y procedimientos que se utilizan en la configuración de los nuevos sistemas de trabajo (Peiró, 2004).

Todos estos planteamientos tienen fuertes implicaciones sobre las actividades laborales y la cualificación de los trabajadores. Determinados conocimientos, habilidades y destrezas que eran importantes en el desempeño del trabajo resultan ahora prácticamente irrelevantes, y competencias que antes ni siquiera se tomaban en consideración resultan ahora imprescindibles (Peiró, 2004). En la tabla 1 se presenta el resumen de la revisión de la literatura. 
Tabla 1

Resumen de la revisión de literatura

\begin{tabular}{|c|c|c|}
\hline Autor & Aportación & Contenido \\
\hline Maslow (1943) & $\begin{array}{l}\text { Teoría de la jerarquía } \\
\text { de las necesidades }\end{array}$ & $\begin{array}{l}\text { La escala de las necesidades se describe como una pirámide } \\
\text { de } 5 \text { niveles: los } 4 \text { primeros niveles pueden ser agrupados } \\
\text { como necesidades primordiales. Al nivel superior lo } \\
\text { denominó autorrealización }\end{array}$ \\
\hline McGregor (1960) & Teoría X y teoría $\mathrm{Y}$ & $\begin{array}{l}\text { Son } 2 \text { teorías contrapuestas de dirección; en la primera, los } \\
\text { directivos consideran que los trabajadores solo actúan bajo } \\
\text { amenazas, y en la segunda, los directivos se basan en el } \\
\text { principio de que la gente quiere y necesita trabajar }\end{array}$ \\
\hline Vroom (1964) & $\begin{array}{l}\text { Teoría de las } \\
\text { expectativas }\end{array}$ & $\begin{array}{l}\text { Un empleado se motiva para ejercer un alto nivel de } \\
\text { esfuerzo cuando cree que ese esfuerzo llevará a una buena } \\
\text { evaluación de su desempeño; una buena evaluación dará } \\
\text { lugar a recompensas organizacionales }\end{array}$ \\
\hline Herzberg (1968) & $\begin{array}{l}\text { Teoría de los } 2 \\
\text { factores }\end{array}$ & $\begin{array}{l}\text { La relación de un individuo con su trabajo es básica, y su } \\
\text { actitud hacia este trabajo bien puede determinar el éxito o el } \\
\text { fracaso del individuo, y esto depende de factores intrínsecos } \\
\text { y extrínsecos }\end{array}$ \\
\hline McClelland (1961) & $\begin{array}{l}\text { Teoría de las } 3 \\
\text { necesidades } \\
\text { secundarias }\end{array}$ & $\begin{array}{l}\text { La motivación de un individuo puede deberse a la búsqueda } \\
\text { de satisfacción de } 3 \text { necesidades dominantes: necesidad de } \\
\text { logro, necesidad de poder y necesidad de afiliación }\end{array}$ \\
\hline Santos (2006) & $\begin{array}{l}\text { Ecología del saber, } \\
\text { emancipación del } \\
\text { conocimiento }\end{array}$ & $\begin{array}{l}\text { En la búsqueda del saber es importante el desaprender, y } \\
\text { más que buscar por igualdades, lo que se requiere es } \\
\text { apreciar las diferencias }\end{array}$ \\
\hline Peiró (2004) & Riesgos psicosociales & $\begin{array}{l}\text { Las transformaciones sociales, económicas y tecnológicas } \\
\text { han generado un nuevo panorama en la actividad de las } \\
\text { empresas }\end{array}$ \\
\hline Bauman (1999) & La sociedad líquida & $\begin{array}{l}\text { El trabajador pasa de un trabajo duradero sin vínculos y } \\
\text { agradecimiento, a un trabajo inmediato en que los vínculos } \\
\text { desaparecen y la gratitud instantánea es lo único que } \\
\text { considera }\end{array}$ \\
\hline Lipovetsky (1998) & La era del vacío & $\begin{array}{l}\text { Vivimos en una era del vacío en la que la sociedad } \\
\text { posmoderna individualista sustentada en valores materiales, } \\
\text { que no encuentra un fin o metas colectivas, solo un fin } \\
\text { individual, lo que no le proporciona ningún tipo de } \\
\text { satisfacción }\end{array}$ \\
\hline
\end{tabular}

Fuente: elaboración propia.

Investigaciones previas han estudiado si los motivadores en el trabajo y los valores organizacionales de las empresas difieren de acuerdo al segmento generacional (Montana y Lenaghan, 1999; Leschinsky y Michael, 2004). Considerando estas investigaciones, este estudio investigó las diferencias existentes entre la importancia de los motivadores en el trabajo y los valores organizacionales en cuanto a la pertenencia generacional de los empleados que trabajan en la industria maquiladora de Ciudad Juárez. Considerando los elementos anteriores, el proceso de diseño de la investigación siguió el diseño recomendado por Cooper y Schindler (2003).

Este diseño hace énfasis en la necesidad de plantear las preguntas de investigación como fase principal y posteriormente efectuar su análisis, el cual definirá en definitiva el objetivo de la investigación. Conforme al diseño mencionado, se plantearon las siguientes preguntas e hipótesis. 
Pregunta 1. ¿En qué medida la importancia de los motivadores en el trabajo de los empleados de la industria maquiladora de Ciudad Juárez se relaciona por la pertenencia a las 4 generaciones?

Hipótesis 1. No existe una diferencia estadísticamente significativa en la importancia de los motivadores en el trabajo entre las 4 generaciones de empleados que trabajan en la industria maquiladora en Ciudad Juárez.

Pregunta 2. ¿En qué medida la importancia de los valores organizacionales de los empleados de la industria maquiladora de Ciudad Juárez tiene una diferencia por la pertenencia a una generación?

Hipótesis 2. No existe una diferencia estadísticamente significativa en la importancia de los valores organizacionales de las 4 generaciones de empleados que trabajan en la industria maquiladora de Ciudad Juárez.

Si la motivación es el problema número uno que enfrentan las organizaciones en el mundo (Hernández, Gallarzo y Espinoza, 2011), entender la diferencia existente, si la hay, en valores y expectativas de una fuerza de trabajo multigeneracional es esencial para el éxito. Diseñar adecuadamente las políticas, los procedimientos y un sistema de recompensas representa un medio adecuado para aumentar la motivación en el trabajo. Depender exclusivamente de las características generacionales de la fuerza laboral actual en las empresas no será suficiente para determinar con precisión la importancia oculta de motivación en el trabajo. Entender los valores y los estereotipos generacionales puede ser un largo camino hacia la obtención de un personal motivado y obtener ventajas competitivas; de no hacerlo, los costos pueden resultar muy altos.

\section{Metodología}

Para la validación de las hipótesis planteadas se realizó una investigación empírica en la industria maquiladora de Ciudad Juárez. El estudio consistió de 2 partes de una encuesta previamente validada que mide, por un lado, la importancia de los motivadores en el trabajo, y por otro lado, la importancia de los valores organizacionales de los encuestados. Las 2 partes fueron combinadas en una sola, en un cuestionario autoadministrado. Este cuestionario consta de 38 ítems validados previamente en las investigaciones de Montana y Lenaghan (1999) y Leschinsky y Michael (2004), para indicar la importancia de 20 motivadores y 18 valores organizacionales.

El método de consistencia interna representa el medible más popular de evaluar el nivel de confiabilidad. El índice más utilizado para medir la consistencia interna de un instrumento es el coeficiente de alfa Cronbach (Cronbach, 1951), el cual es usado en este estudio. El valor de alfa Cronbach puede ser entre 0 y 1 , y depende del promedio de la correlación entre los elementos utilizados en el instrumento y el número de elementos utilizados en la escala. Un investigador trata de medir una cualidad no directamente observable en una población de sujetos. Para ello mide $n$ variables que sí son observables de cada uno de los sujetos.

Se supone que las variables están relacionadas con la magnitud inobservable de interés. En particular, las $n$ variables deberían realizar mediciones estables y consistentes, con un elevado nivel de correlación entre ellas. El alfa de Cronbach permite cuantificar el nivel de fiabilidad de una escala de medida para la magnitud inobservable construida a partir de las $n$ variables observadas. Los valores del índice alfa Cronbach arriba de 0.70 son esenciales para considerarse válidos.

La confiabilidad en un índice alfa de Cronbach para los instrumentos utilizados alcanza 0.72 en los 20 ítems de los motivadores y 0.78 en los ítems de evaluación de los valores organizacionales. La validación de constructo del cuestionario se llevó a cabo a través de la revisión del marco teórico existente relacionado a los 20 motivadores y 18 valores organizacionales. La validez de 
Tabla 2

Distribución de los empleados que trabajan en las empresas maquiladoras de acuerdo al IMSS

\begin{tabular}{lcc}
\hline Generación (período) & Total empleados & Porcentaje \\
\hline Tradicionalistas (antes de 1946) & 12,751 & $3.7 \%$ \\
Baby boomers (1946-1964) & 140,605 & $40.8 \%$ \\
Generación X (1965-1980) & 118,894 & $34.5 \%$ \\
Generación Y (1981-2000) & 72,371 & $21.0 \%$ \\
Total & 344,621 & $100 \%$ \\
\hline
\end{tabular}

Fuente: IMSS. Distribución de las 4 generaciones de empleados que trabajan en la industria maquiladora en Ciudad Juárez en el mes de abril del 2013.

contenido se realizó evaluando si los elementos del cuestionario eran relevantes para la medición del contenido de los motivadores y valores organizacionales. La validez de la muestra se determinó para establecer qué tan bien la proporción del total de la población está siendo probada. Una prueba piloto a 30 empleados de maquiladora, diferentes a los encuestados en este estudio, fue realizada para determinar la validez de contenido del cuestionario utilizado en este estudio.

En 1966 Ciudad Juárez fue el asentamiento de las primeras maquiladoras, y con ello se desarrolló el primer Parque Industrial en México, el Parque Bermúdez, muy al estilo de los parques industriales estadounidenses y con los requerimientos establecidos por ellos. Ciudad Juárez, como otras ciudades de la frontera, presentó una serie de elementos que la hicieron atractiva para la instalación de las plantas maquiladoras, entre ellas la gran oferta de mano de obra calificada y su proximidad geográfica con Estados Unidos, que disminuyó los costos de transporte entre las plantas y además permitió que el personal estadounidense viviera en su país y viajara a diario hasta su lugar de trabajo (CEPAL, 1996).

Como tendencia general, las ciudades del mundo, en la primera década del siglo XXI, se están enfrentando a un fenómeno de reestructuración que varios estudiosos han coincidido en llamar el ascenso de la ciudad global o la ciudad mundial de la era de la información (Borja y Castells, 1997). Ciertos rasgos, como la presencia de fuerte inversión extranjera y los movimientos de capital, el incremento en la producción de servicios avanzados, la localización abundante de oficinas principales, la incorporación en los flujos de información, la reorientación de las tendencias migratorias y la aguda polarización social que se advierten desde las últimas décadas, revelan que, desde el establecimiento de la industria maquiladora en su territorio, Ciudad Juárez se ha consolidado como vínculo articulador de la economía del país con la economía mundial, constatando de esta forma su integración en los procesos de globalización. A ello, ahora habrá que añadirle el ingrediente de la confluencia de 4 generaciones de empleados en las empresas. En datos de abril del 2013, los empleados registrados en el Seguro Social que trabajaban en la industria maquiladora de Ciudad Juárez ascendían a 344,621 personas.

La tabla 2 muestra que el grupo de edad nacidos antes de 1946, correspondiente a la generación tradicionalista, es por mucho el grupo más pequeño y seguirá reduciéndose como miembros de un grupo en edad de retiro. Los otros 3 grupos corresponden a las generaciones de los baby boomers, generación X y generación $\mathrm{Y}$, por lo que la fuerza de trabajo estará compuesta casi por igual por miembros de estas 3 generaciones, quienes habrán de competir en condiciones de igualdad con un mayor o menor rol buscando cubrir sus propias necesidades.

El «diseño total» de Dillman (2000) fue utilizado como técnica de envíos de correos electrónicos. Dillman sugiere personalizar cada correo electrónico en lugar de enviar los correos a todos los encuestados a la vez. La utilización de este método permite obtener las respuestas de 4 a 5 semanas, a diferencia del método tradicional, que se lleva de 8 a 10 semanas. 
Tabla 3

Administración de los cuestionarios enviados

\begin{tabular}{lcc}
\hline Total de correos por categoría de respuesta & Frecuencia & Cálculo \\
\hline Tamaño de la muestra inicial & 2,538 & \\
Notificaciones de no envío & 423 & \\
Declinaron participar & 479 & \\
Tamaño de la muestra efectivo & 2,114 & $2,538-(423+15)$ \\
Respuestas completas & 1,271 & $1,271 / 2,114$ \\
Tasa de respuesta & $60.12 \%$ & \\
\hline
\end{tabular}

Fuente: elaboración propia

Tabla 4

Distribución de encuestados por generación

\begin{tabular}{lcc}
\hline Generación & Empleados & Porcentaje \\
\hline Tradicionalistas: 65-74 años & 52 & $4.09 \%$ \\
Baby boomers: 45-64 años & 483 & $38.0 \%$ \\
Generación X: 30-44 años & 458 & $36.03 \%$ \\
Generación Y: 21-29 años & 278 & $21.88 \%$ \\
Total & 1,271 & $100 \%$ \\
\hline
\end{tabular}

Fuente: elaboración propia.

Tabla 5

Distribución de encuestados por giro de la empresa

\begin{tabular}{lcc}
\hline Giro & Empleados & Porcentaje \\
\hline Automotriz & 464 & $36.5 \%$ \\
Electrónico & 627 & $49.33 \%$ \\
Médico & 180 & $14.17 \%$ \\
Total & 1,271 & $100 \%$ \\
\hline
\end{tabular}

Fuente: elaboración propia.

Se tuvieron 2,538 direcciones de correo electrónico, y para ello se utilizaron los directorios empresariales y de la Asociación de Maquiladoras. Durante la primera semana se recibieron respuestas de 185 encuestados; 423 correos electrónicos fueron regresados por el sistema sin poder entregarlos. Se recibieron 48 respuestas automáticas de «out of office», y 479 pidieron ser removidos de la lista de encuestados por políticas de la empresa. De los seleccionados, 1,271 completaron y regresaron la encuesta (tabla 3).

De los 1,271 encuestados que devolvieron el instrumento contestado completo, el mayor porcentaje pertenece a los baby boomers, seguidos de la generación X (tabla 4). Los encuestados con menor porcentaje de respuesta fueron los tradicionalistas, siendo este dato consistente con la información recabada en el Instituto Mexicano del Seguro Social sobre el porcentaje de empleados por generación (tabla 4).

En la tabla 5, de los empleados encuestados el $49.33 \%$ correspondieron a trabajadores que pertenecen a empresas del ramo electrónico. El menor porcentaje, con un $14.17 \%$, fueron los trabajadores en empresas del giro médico. Los empleados que devolvieron la encuesta completa pertenecen a diferentes niveles y categorías dentro de las empresas. En estas categorías se encuadraron empleados administrativos, supervisores y gerentes. De estos empleados encuestados, el $35.8 \%$ fueron mujeres. A todos los empleados encuestados se les requirió tener al menos nivel 
Tabla 6

Estadística demográfica de los empleados encuestados

\begin{tabular}{lll}
\hline Variable & & \\
\hline$n$ & & 1,271 \\
Género & Hombres & 64.2 \\
$\quad \%$ & Mujeres & 35.8 \\
$\%$ & & \\
Nivel de educación & Licenciatura & 78 \\
$\quad \%$ & Maestría & 22 \\
$\%$ & & 44 \\
Media edad (años) & & 8.3 \\
Media experiencia (años) & & \\
Edad & & 19 \\
$\quad \%$ & $20-30$ & 34 \\
$\%$ & $31-40$ & 21 \\
$\%$ & $41-50$ & 26 \\
Categoría & $51+$ & 28 \\
$\%$ & & 65 \\
$\%$ & Empleados administrativos & \\
\hline
\end{tabular}

Fuente: elaboración propia.

Tabla 7

Ejemplo de las preguntas y la escala utilizada en el instrumento

\begin{tabular}{lllll}
\hline \multicolumn{2}{c}{ Por favor, indique la importancia de los siguientes elementos en términos de que tanto lo motivan a usted para hacer } \\
cumplir con su trabajo en su actual empleo
\end{tabular}

Fuente: elaboración propia.

de licenciatura para participar en el estudio. El promedio de edad fue de 44 años. La estadística descriptiva de la información demográfica se presenta en la tabla 6.

Adicionalmente, la tabla 5 muestra la distribución de encuestados por giro de la empresa.

El instrumento utilizado en este estudio evalúa la importancia de 20 motivadores en el trabajo y 18 valores organizacionales. El cuestionario utiliza una escala de Likert de 5 puntos desde 1 (no importante) hasta 5 (extremadamente importante). La tabla 7 muestra un ejemplo de las preguntas planteadas. La primera página explicó el propósito del estudio, el tiempo estimado para completar el cuestionario, la confidencialidad de los datos y la información de contacto para información adicional. 
Tabla 8

Operacionalización de las variables

\begin{tabular}{|c|c|c|c|c|}
\hline Variable & Dimensión & Prueba estadística & $\begin{array}{l}\text { Diferencias en } \\
\text { medias }\end{array}$ & $\begin{array}{l}\text { Factores } \\
\text { extraídos }\end{array}$ \\
\hline Ver tabla 9 & Motivadores & $\begin{array}{l}\text { ANOVA } \\
\text { Análisis factorial } \\
\text { Rotación de Oblimin }\end{array}$ & Prueba t & $\begin{array}{l}\text { Prueba de } \\
\text { Bonferroni }\end{array}$ \\
\hline Ver tabla 11 & $\begin{array}{l}\text { Valores orga- } \\
\text { nizacionales }\end{array}$ & ANOVA & Prueba t & $\begin{array}{l}\text { Prueba de } \\
\text { Bonferroni }\end{array}$ \\
\hline
\end{tabular}

Fuente: elaboración propia.

\section{Resultados}

Para analizar la existencia de posibles relaciones estadísticamente significativas entre las 4 generaciones en los motivadores de trabajo percibidos, valores ideales y percepciones de cómo las empresas maquiladoras demuestran los valores organizacionales con el ideal, se realizó un análisis de varianza (ANOVA). Cuando se encontraron diferencias, se realizaron pruebas t independientes entre cada grupo para determinar las diferencias en los ítems de la encuesta.

Para evitar el problema de comparación múltiple que se presentó al realizar varias pruebas t, se utilizó la técnica de Bonferonni para controlar el error tipo I en cada uno de los individuos analizados. Adicionalmente, los datos recolectados fueron analizados por medio de un análisis factorial de componentes principales. Se utilizó el análisis factorial para reducir el número de elementos a un conjunto más pequeño que podrían utilizarse para entender patrones en los puntos de la encuesta. La operacionalización de las variables se muestra en la tabla 8.

Un análisis de las variables que tuvieron más peso se determinó para derivar los datos y el número de factores. El siguiente análisis incluye los resultados de medir la importancia en los 20 motivadores en el trabajo y la importancia de los 18 valores organizacionales relativos a las elecciones de los participantes de los ideales de las empresas. Dado que uno de los requisitos para que el análisis factorial tenga sentido es que las variables estén altamente correlacionadas, considerando las 2 dimensiones del cuestionario y los antecedentes en las variables involucradas, se procedió solamente a realizar análisis factorial en las variables de la dimensión de motivadores. Los resultados obtenidos se muestran en la tabla 9.

La hipótesis nula 1 planteó:

No existen diferencias estadísticamente significativas en la preferencia de los motivadores en el trabajo entre las 4 generaciones de los empleados que trabajan en la industria maquiladora en Ciudad Juárez. La tabla 9 presenta la media, la desviación estándar y la significancia estadística de cada uno de los motivadores utilizados en el instrumento y sus diferencias entre generaciones.

Considerando los resultados obtenidos, la hipótesis nula de que no existe una diferencia estadísticamente significativa en las preferencias de los motivadores del trabajo entre las 4 generaciones de empleados que trabajan en la industria maquiladora de Ciudad Juárez puede ser rechazada. Las pruebas $\mathrm{t}$ realizadas revelaron que tanto la generación $\mathrm{X}(\mathrm{p}<0.006)$ como la generación $\mathrm{Y}$ $(\mathrm{p}<0.001)$ consideran como un motivador los tiempos de comida, a diferencia de los participantes de los tradicionalistas y los baby boomers.

En cuanto a un buen sueldo, la generación X considera estadísticamente $(\mathrm{p}=0.000)$ este aspecto como un motivador más fuerte en comparación a como lo consideran los baby boomers $(\mathrm{p}<0.001)$ y los tradicionalistas $(\mathrm{p}=0.002)$. Las buenas condiciones de trabajo son consideradas tanto por 
Tabla 9

Importancia de los motivadores en el trabajo para las 4 generaciones que trabajan en la industria maquiladora

\begin{tabular}{|c|c|c|c|c|c|c|c|}
\hline Motivadores & & $\begin{array}{l}\text { Tradicionalistas } \\
(\mathrm{n}=52)\end{array}$ & $\begin{array}{l}\text { Baby boomers } \\
(\mathrm{n}=483)\end{array}$ & $\begin{array}{l}\text { Generación } X \\
(n=458)\end{array}$ & $\begin{array}{l}\text { Generación Y } \\
(\mathrm{n}=278)\end{array}$ & Sig. & $\begin{array}{l}\text { Diferencias en } \\
\text { medias a un } \\
\text { nivel de } 0.05\end{array}$ \\
\hline \multirow{2}{*}{$\begin{array}{l}\text { Tener estabilidad en el } \\
\text { empleo }\end{array}$} & $\mu$ & 5.98 & 6.14 & 6.05 & 6.08 & 0.333 & \\
\hline & $\sigma$ & 1.22 & 1.20 & 1.13 & 1.28 & & \\
\hline \multirow[t]{2}{*}{$\begin{array}{l}\text { Mi supervisor me respeta } \\
\text { como persona }\end{array}$} & $\mu$ & 5.90 & 6.10 & 6.11 & 6.09 & 0.982 & \\
\hline & $\sigma$ & 1.10 & 1.12 & 1.13 & 1.08 & & \\
\hline \multirow{2}{*}{$\begin{array}{l}\text { Son adecuados los tiempos } \\
\text { para comidas }\end{array}$} & $\mu$ & 3.74 & 3.72 & 4.07 & 4.42 & 0.001 & $\mathrm{~d}, \mathrm{e}$ \\
\hline & $\sigma$ & 1.40 & 1.52 & 1.55 & 1.65 & & \\
\hline \multirow[t]{2}{*}{ Buen sueldo } & $\mu$ & 5.63 & 5.90 & 6.18 & 6.10 & 0.001 & $\mathrm{~b}, \mathrm{~d}$ \\
\hline & $\sigma$ & 1.10 & 1.00 & 0.95 & 1.02 & & \\
\hline \multirow{2}{*}{$\begin{array}{l}\text { Condiciones físicas de } \\
\text { trabajo buenas }\end{array}$} & $\mu$ & 5.30 & 5.32 & 5.33 & 4.93 & 0.02 & $\mathrm{e}, \mathrm{f}$ \\
\hline & $\sigma$ & 1.07 & 1.32 & 1.30 & 1.40 & & \\
\hline \multirow{2}{*}{$\begin{array}{l}\text { Oportunidad de producir un } \\
\text { trabajo de calidad }\end{array}$} & $\mu$ & 6.39 & 6.35 & 6.23 & 5.93 & 0.001 & $\mathrm{c}, \mathrm{e}$ \\
\hline & $\sigma$ & 0.88 & .86 & 0.90 & 1.04 & & \\
\hline \multirow{2}{*}{$\begin{array}{l}\text { Llevarse bien con otros en } \\
\text { el trabajo }\end{array}$} & $\mu$ & 5.58 & 5.53 & 5.36 & 5.36 & 0.305 & \\
\hline & $\sigma$ & 1.10 & 1.29 & 1.36 & 1.35 & & \\
\hline \multirow{2}{*}{$\begin{array}{l}\text { Oportunidad de } \\
\text { promociones }\end{array}$} & $\mu$ & 5.31 & 5.11 & 6.11 & 6.21 & 0.001 & $\mathrm{a}, \mathrm{b}, \mathrm{c}, \mathrm{d}$ \\
\hline & $\sigma$ & 1.44 & 1.35 & 1.19 & 1.16 & & \\
\hline \multirow{2}{*}{$\begin{array}{l}\text { Oportunidad de hacer } \\
\text { trabajo interesante }\end{array}$} & $\mu$ & 6.41 & 5.97 & 6.05 & 6.10 & 0.085 & \\
\hline & $\sigma$ & 0.76 & 1.12 & 1.14 & 1.18 & & \\
\hline \multirow{2}{*}{$\begin{array}{l}\text { Pensión y beneficios de } \\
\text { seguridad social }\end{array}$} & $\mu$ & 5.79 & 5.97 & 5.57 & 5.40 & 0.001 & d,e \\
\hline & $\sigma$ & 1.15 & 1.13 & 1.36 & 1.42 & & \\
\hline \multirow{2}{*}{$\begin{array}{l}\text { Ser apreciado por mi } \\
\text { supervisor }\end{array}$} & $\mu$ & 5.88 & 5.68 & 5.65 & 5.54 & 0.402 & \\
\hline & $\sigma$ & 1.04 & 1.17 & 1.21 & 1.29 & & \\
\hline \multirow{2}{*}{$\begin{array}{l}\text { Siento que mi trabajo es } \\
\text { importante }\end{array}$} & $\mu$ & 6.18 & 5.79 & 5.78 & 5.67 & 0.189 & \\
\hline & $\sigma$ & 0.95 & 1.17 & 1.12 & 1.34 & & \\
\hline
\end{tabular}


Tabla 9 (continuación)

\begin{tabular}{|c|c|c|c|c|c|c|c|}
\hline Motivadores & & $\begin{array}{l}\text { Tradicionalistas } \\
(\mathrm{n}=52)\end{array}$ & $\begin{array}{l}\text { Baby boomers } \\
(\mathrm{n}=483)\end{array}$ & $\begin{array}{l}\text { Generación } X \\
(\mathrm{n}=458)\end{array}$ & $\begin{array}{l}\text { Generación Y } \\
(\mathrm{n}=278)\end{array}$ & Sig. & $\begin{array}{l}\text { Diferencias en } \\
\text { medias a un } \\
\text { nivel de } 0.05\end{array}$ \\
\hline \multirow{2}{*}{ Tener variedad en mis tareas } & $\mu$ & 5.55 & 5.24 & 5.30 & 5.51 & 0.078 & \\
\hline & $\sigma$ & 1.36 & 1.28 & 1.25 & 1.35 & & \\
\hline \multirow{2}{*}{$\begin{array}{l}\text { Ser felicitado por mi jefe } \\
\text { por mi buen trabajo }\end{array}$} & $\mu$ & 4.94 & 4.75 & 4.98 & 4.97 & 0.298 & \\
\hline & $\sigma$ & 1.26 & 1.45 & 1.67 & 1.68 & & \\
\hline \multirow{2}{*}{$\begin{array}{l}\text { Obtener una evaluación de } \\
\text { desempeño }\end{array}$} & $\mu$ & 4.70 & 4.38 & 4.48 & 4.39 & 0.506 & \\
\hline & $\sigma$ & 1.55 & 1.71 & 1.75 & 1.73 & & \\
\hline \multirow{2}{*}{$\begin{array}{l}\text { Sé que soy una diferencia } \\
\text { en la empresa }\end{array}$} & $\mu$ & 6.21 & 5.70 & 5.45 & 5.33 & 0.001 & $\mathrm{~b}, \mathrm{c}, \mathrm{e}$ \\
\hline & $\sigma$ & 1.14 & 1.19 & 1.41 & 1.38 & & \\
\hline \multirow{2}{*}{$\begin{array}{l}\text { Oportunidad para mejorar } \\
\text { mis habilidades }\end{array}$} & $\mu$ & 5.49 & 5.66 & 5.95 & 6.07 & 0.001 & $\mathrm{c}, \mathrm{d}, \mathrm{e}$ \\
\hline & $\sigma$ & 1.05 & 1.16 & 1.10 & 1.15 & & \\
\hline \multirow[t]{2}{*}{ Vacaciones razonables } & $\mu$ & 5.25 & 5.44 & 5.51 & 5.69 & 0.062 & \\
\hline & $\sigma$ & 1.19 & 1.29 & 1.35 & 1.26 & & \\
\hline \multirow{2}{*}{$\begin{array}{l}\text { Sé que tendrá consecuencias } \\
\text { si hago un mal trabajo }\end{array}$} & $\mu$ & 3.64 & 3.81 & 4.14 & 4.13 & 0.071 & \\
\hline & $\sigma$ & 1.72 & 1.67 & 1.71 & 1.60 & & \\
\hline \multirow{2}{*}{$\begin{array}{l}\text { Libertad amplia en el } \\
\text { trabajo }\end{array}$} & $\mu$ & 5.77 & 5.50 & 5.39 & 5.24 & 0.085 & \\
\hline & $\sigma$ & 1.45 & 1.23 & 1.25 & 1.28 & & \\
\hline
\end{tabular}

${ }^{a}$ Diferencias entre tradicionalistas y baby boomers.

b Diferencias entre tradicionalistas y generación X.

c Diferencias entre tradicionalistas y generación $\mathrm{Y}$.

d Diferencias entre baby boomers y generación X.

e Diferencias entre baby boomers y generación Y.

${ }^{\mathrm{f}}$ Diferencias entre generación X y generación Y.

La prueba de Bonferroni tuvo un nivel alfa de 0.005.

Fuente: elaboración propia. 
los baby boomers como los miembros de la generación X como un alto motivador en comparación con los de la generación Y, que no lo consideran un gran motivador.

En cuanto a las oportunidades de promoción, las pruebas revelaron que los tradicionalistas y los baby boomers no lo consideran un factor de motivación, en comparación con los participantes de la generación X $(\mathrm{p}<0.001)$ y los de la generación Y $(\mathrm{p}<0.001)$. Por el contrario, la pensión y otros beneficios fueron uno de los más altos motivadores en comparación con los grupos de la generación $\mathrm{X}(\mathrm{p}=0.000)$ y la generación $\mathrm{Y}(\mathrm{p}=.001)$.

Ser una diferencia en la empresa reveló mediante la prueba realizada que los tradicionalistas lo califican como uno de los más grandes motivadores, cuando se comparan con la generación $\mathrm{X}$ $(\mathrm{p}=0.001)$ y la generación $\mathrm{Y}(\mathrm{p}=0.001)$. Adicionalmente, para el grupo de la generación $\mathrm{Y}$ la oportunidad para mejorar sus habilidades calificó como uno de los motivadores más altos al compararse con los tradicionalistas $(\mathrm{p}=0.005)$ y los baby boomers $(\mathrm{p}=0.001)$. La generación $\mathrm{X}$ también lo consideró como un más alto motivador en comparación con los baby boomers $(\mathrm{p}=0.001)$.

Los elementos de la pregunta 1 también se sometieron a un análisis factorial de componentes principales. El método utilizado por defecto en la mayoría de los paquetes informáticos es la rotación varimax, que es un método de rotación ortogonal que minimiza el número de variables que tienen saturaciones altas en cada factor. Simplifica la interpretación de los factores y tiende a producir grupos de factores múltiples; Sin embargo, el mantenimiento de ortogonalidad a menudo dificulta su interpretación. Al exigir la ortogonalidad, estamos imponiendo que las variables no tienen nada que ver unas con otras, y esto en la práctica parece difícilmente sostenible. Por eso tiene más interés hacer uso de métodos de rotación oblicuos. El más común entre estos últimos es el método Oblimin. Este método tiende a producir las mismas soluciones que la rotación varimax pero permite que sean oblicuas, lo que facilita su interpretación y su uso práctico.

Los factores resultantes se giraron utilizando la rotación de Oblimin a fin de ayudar en la interpretación de los factores. Este análisis de componentes principales dio lugar a 5 factores extraídos que tenían un valor propio mayor que uno. Cada uno de los 5 factores fue interpretado y ayudan explicar la estructura factorial subyacente de la encuesta con los 18 motivadores en el trabajo. Los 5 factores explican un total 63,28\% de la varianza. Los siguientes resultados presentan una interpretación y posteriormente un análisis que señala las diferencias entre los 4 grupos en los factores extraídos. La tabla 10 muestra los resultados de las pruebas realizadas.

Las pruebas realizadas muestran que para los baby boomers la seguridad en el empleo es un factor motivador si se compara con el grupo de la generación $\mathrm{Y}(\mathrm{p}=0.001)$; sin embargo, los baby boomers mostraron el factor de retroalimentación en el desempeño como menos motivador en relación a la generación $\mathrm{X}(\mathrm{p}<0.001)$ y la generación $\mathrm{Y}(\mathrm{p}=0.001)$. El análisis realizado mostró que no existen diferencias significativas en el factor de seguridad en el empleo y el factor de retroalimentación en el desempeño, en función del grupo generacional.

La hipótesis nula 2 planteó:

No existen diferencias estadísticamente significativas en las preferencias de los valores organizacionales de las 4 generaciones de empleados que trabajan en la industria maquiladora de Ciudad Juárez.

Los resultados de las pruebas que evalúan las diferencias de los valores organizacionales de los 4 grupos generacionales, medidos mediante la prueba ANOVA, resultaron significativos. La información obtenida establece que la prueba ANOVA fue significativa para 4 elementos: predicción, oportunidad de crecer profesionalmente, estar orientado a las reglas y no ser restringido por reglas. La tabla 11 muestra los resultados de las pruebas realizadas.

Considerando los resultados obtenidos, la hipótesis nula planteada puede ser rechazada. Los resultados muestran diferencias entre los grupos generacionales. El aspecto de predicción muestra 
Tabla 10

Prueba de los factores extraídos del análisis de los componentes principales de los motivadores en el trabajo

\begin{tabular}{|c|c|c|c|c|c|c|c|}
\hline Factores & & $\begin{array}{l}\text { Tradicionalistas } \\
(\mathrm{n}=50)\end{array}$ & $\begin{array}{l}\text { Baby boomers } \\
(\mathrm{n}=463)\end{array}$ & $\begin{array}{l}\text { Generación } X \\
(\mathrm{n}=437)\end{array}$ & $\begin{array}{l}\text { Generación } Y \\
(\mathrm{n}=266)\end{array}$ & Sig. & $\begin{array}{l}\text { Diferencias en } \\
\text { medias a un } \\
\text { nivel de } 0.05\end{array}$ \\
\hline \multirow[t]{2}{*}{ Sentirme apreciado e importante } & $\mu$ & 34.90 & 33.32 & 33.01 & 32.67 & 0.148 & \\
\hline & $\sigma$ & 5.24 & 5.41 & 5.75 & 5.97 & & \\
\hline \multirow[t]{2}{*}{ Seguridad en el empleo } & $\mu$ & 11.68 & 12.02 & 11.64 & 11.42 & 0.003 & $\mathrm{e}$ \\
\hline & $\sigma$ & 2.06 & 1.89 & 2.13 & 2.28 & & \\
\hline \multirow{2}{*}{$\begin{array}{l}\text { Habilidades y experiencia en el } \\
\text { trabajo }\end{array}$} & $\mu$ & 30.06 & 28.84 & 29.10 & 29.17 & 0.351 & \\
\hline & $\sigma$ & 3.36 & 3.88 & 4.05 & 4.5 & & \\
\hline \multirow[t]{2}{*}{ Condiciones de trabajo } & $\mu$ & 19.90 & 19.91 & 20.20 & 20.29 & 0.598 & \\
\hline & $\sigma$ & 3.81 & 3.60 & 3.90 & 3.92 & & \\
\hline \multirow[t]{2}{*}{ Retroalimentación del desempeño } & $\mu$ & 19.19 & 19.18 & 20.87 & 20.85 & 0.001 & $\mathrm{~d}, \mathrm{e}$ \\
\hline & $\sigma$ & 3.87 & 4.20 & 3.95 & 3.91 & & \\
\hline
\end{tabular}

d Diferencias entre baby boomers y generación X.

e Diferencias entre baby boomers y generación Y.

La prueba de Bonferonni tuvo un nivel alfa de 0.005.

Fuente: elaboración propia. 
Tabla 11

ANOVA y prueba t de las preferencias en los valores organizacionales de los 4 grupos generacionales

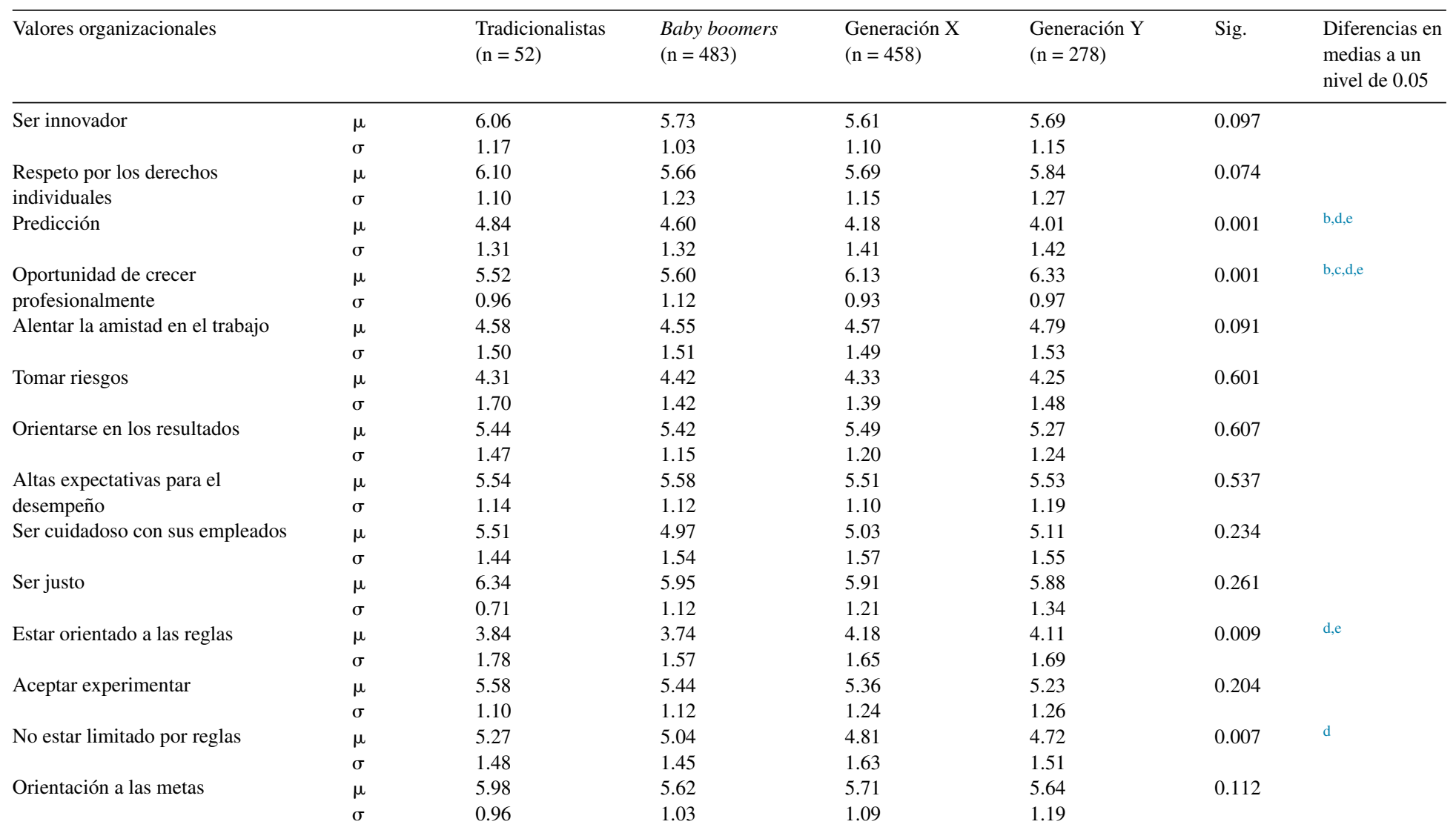


Tabla 11 (continuación)

\begin{tabular}{|c|c|c|c|c|c|c|c|}
\hline Valores organizacionales & & $\begin{array}{l}\text { Tradicionalistas } \\
(\mathrm{n}=52)\end{array}$ & $\begin{array}{l}\text { Baby boomers } \\
(\mathrm{n}=483)\end{array}$ & $\begin{array}{l}\text { Generación } X \\
(\mathrm{n}=458)\end{array}$ & $\begin{array}{l}\text { Generación } Y \\
(\mathrm{n}=278)\end{array}$ & Sig. & $\begin{array}{l}\text { Diferencias en } \\
\text { medias a un } \\
\text { nivel de } 0.05\end{array}$ \\
\hline Obtener ventaja de las & $\mu$ & 5.59 & 5.58 & 5.67 & 5.71 & 0.727 & \\
\hline oportunidades & $\sigma$ & 1.41 & 1.09 & 1.16 & 1.19 & & \\
\hline \multirow[t]{2}{*}{ Tolerancia } & $\mu$ & 5.39 & 4.98 & 5.05 & 4.99 & 0.298 & \\
\hline & $\sigma$ & 1.49 & 1.31 & 1.39 & 1.38 & & \\
\hline \multirow[t]{2}{*}{ Estabilidad } & $\mu$ & 5.72 & 5.88 & 5.83 & 5.87 & 0.901 & \\
\hline & $\sigma$ & 1.13 & 1.12 & 1.26 & 1.29 & & \\
\hline \multirow[t]{2}{*}{ Seguridad en el empleo } & $\mu$ & 5.91 & 6.08 & 6.10 & 6.22 & 0.423 & \\
\hline & $\sigma$ & 1.05 & 1.04 & 1.18 & 1.16 & & \\
\hline
\end{tabular}

b Diferencias entre tradicionalistas y generación $X$.

c Diferencias entre tradicionalistas y generación Y.

d Diferencias entre baby boomers y generación X.

e Diferencias entre baby boomers y generación Y.

La prueba de Bonferonni tuvo un nivel alfa de 0.007.

Fuente: elaboración propia. 
que el grupo tradicionalista lo considera más deseable que la generación $X(p=0.001)$. Incluso la generación de los baby boomers califican este valor más alto tanto para la generación $\mathrm{X}(\mathrm{p}=0.001)$ y la generación Y $(\mathrm{p}=0.001)$.

Respecto a proporcionar oportunidades para el crecimiento, la generación X y la generación $\mathrm{Y}$ califican $(\mathrm{p}=0.001)$ más alto en este aspecto que los grupos pertenecientes a generación de los tradicionalistas y los baby boomers. En referencia a estar orientado a las reglas, la generación X e Y evalúan más alto $(\mathrm{p}=0.005)$ que los baby boomers. Finalmente, el no estar restringido por reglas es un aspecto que los baby boomers evalúan más alto que las generaciones $\mathrm{X}(\mathrm{p}=0.002 \mathrm{e}$ $\mathrm{Y}(\mathrm{p}=0.004)$.

\section{Discusión}

Los resultados de este estudio apoyan los resultados de investigaciones anteriores referentes a que existen diferencias significativas entre las generaciones en la importancia de motivadores de trabajo y valores de la empresa. Esto es consistente con las afirmaciones de Peiró (2004), de que las transformaciones sociales, económicas y tecnológicas han generado un nuevo panorama en la actividad de las empresas. Sin embargo, el alto grado de homogeneidad en las percepciones de los valores de la empresa entre los ideales de las generaciones sugiere otro enfoque en la investigación generacional, así como de la organización. A este respecto es importante lo que menciona Santos (2006), que lo importante no es la homogeneización sino las diferencias iguales.

Las diferencias más significativas en los motivadores se presentaron en el aspecto de considerarse una diferencia en la empresa, la oportunidad para promociones y la oportunidad para aprovechar las habilidades en las distintas generaciones. La seguridad en el empleo fue la variable en que se dieron las diferencias significativas entre baby boomers y la generación Y. En cuanto a los valores organizacionales, la oportunidad para crecer profesionalmente fue la variable en que se obtuvo una diferencia estadísticamente significativa entre las 4 generaciones.

Comprender las diferencias entre generaciones en sus preferencias en los motivadores de trabajo y valores de la empresa tiene también aplicaciones prácticas. Así, en concordancia con Santos (2006), lo que se requiere hoy en día es una ecología de saberes, esto es, desaprender, crear una nueva emancipación del conocimiento que parta de nuevas formas de actuar y de hacer las cosas. Al menos, la comprensión de estas diferencias puede ayudar a las organizaciones a crear mejores estrategias de contratación y retención del talento que se dirijan a las preferencias de cada generación. En este aspecto resulta fundamental tomar en consideración la realidad sociotécnica del sistema de trabajo y el valor de una gestión de los recursos humanos inspirada en los principios humanistas, tal como afirma Peiró (2004). En medio de la evolución de las organizaciones no se puede seguir considerando que las personas no evolucionan. Los sistemas han cambiado, y la importancia en los motivadores y en los valores organizacionales de las diferentes generaciones también lo ha hecho.

Por tanto, las estrategias de contratación centradas en motivadores específicos de viejas o más nuevas generaciones resultan fuera de toda practicidad. Se requiere el análisis de las características generacionales, y a partir de ello establecer nuevas estrategias en las organizaciones. Estas estrategias pueden implicar tanto la comunicación orientada sobre las políticas y los valores que la empresa quiere poner de relieve, como la revisión de las políticas y los recursos para ofrecer entornos de trabajo más estrechamente alineados con las preferencias generacionales. 
Este estudio muestra que entre los empleados de las generaciones X e Y, las oportunidades para promoción y buena paga son los motivadores más altos, por lo que este resultado parece apoyar lo que Lipovetsky (1990) señala al afirmar que el mundo actual vive en una era del vacío sustentado en valores materiales, que no encuentra un fin o metas colectivas, solo un fin individual, lo que no le proporciona ningún tipo de satisfacción. De la misma manera, apoyan la posición de Bauman (1999) de que los trabajadores han migrado de un trabajo duradero, sin vínculos y gratitud, a un trabajo en el que los vínculos desaparecen y el estímulo instantáneo es lo único que considera. En contradicción con la teoría de McClelland, la necesidad de afiliación en las generaciones X e Y empieza a perderse.

Entre los baby boomers y los tradicionalistas, en este estudio las 2 generaciones comparten la oportunidad de producir un trabajo de calidad, calificado primero y segundo entre los factores de motivación, respectivamente, como un motivador superior. Este dato es consistente con la teoría de Herzberg de que existen motivadores intrínsecos y extrínsecos que pueden determinar el éxito o el fracaso de un individuo. En este caso, las organizaciones pueden realizar cambios cuando sea necesario para garantizar los recursos adecuados, incluyendo el tiempo, para promover los resultados de calidad. Adicionalmente, estas 2 mismas generaciones tienen altas expectativas para el desempeño. Las organizaciones pueden recurrir a todas las generaciones mediante la definición de sus expectativas de desempeño para incluir la calidad del trabajo y después para asegurar que los recursos estén disponibles, para que los empleados puedan realizar un trabajo de calidad.

Conforme a la discusión anterior, hoy se vuelve más importante considerar dentro de las organizaciones construir lo que Santos (2006) llama la emancipación del conocimiento, partiendo principalmente de una nueva relación entre el respeto de la igualdad y el principio del reconocimiento de la diferencia. Las organizaciones, al igual que la teoría, se enfocaron en el principio de la igualdad, no por el principio del reconocimiento de las diferencias. Ahora tenemos que intentar construir organizaciones en que tanto la igualdad como las diferencias estén presentes, y saber que una lucha por la igualdad tiene que ser también una lucha por el reconocimiento de la diferencia, porque lo importante no es la homogeneización sino las diferencias iguales.

\section{Conclusiones}

Los resultados obtenidos en este estudio demuestran que los motivadores y la importancia que dan cada una de las generaciones de empleados que trabajan actualmente en las empresas son significativos en el desempeño de sus empleados y en la consecución de metas y objetivos organizacionales.

Dada la homogeneidad de las puntuaciones de los valores preferidos, las administraciones de las empresas maquiladoras pueden ser capaces de igualar sus valores reales de la empresa a los deseados por los empleados que trabajan en la industria. Con frecuencia, las organizaciones que comunican estos valores pueden hacer un mejor trabajo al atraer, contratar y retener a los empleados que se inspiran en los valores con los que se identifican (Chatman, 1991; Vandenberghe y Peiro, 1999).

Las empresas maquiladoras podrían asegurarse que sus estructuras salariales de sus jóvenes empleados son competitivas en el mercado y también para asegurar que se proporciona la formación de conocimientos y habilidades adecuadas para que estos jóvenes sigan siendo competitivos internamente para cualquier promoción. 
Este tipo de industria global se desarrolla en los polos capitalistas y trasciende en los espacios geográficos que mediante su presencia en plantas industriales se encuentran ubicados en los territorios geográficos del subdesarrollo, donde se conjugan valores generacionales de trabajadores con los valores gerenciales globales de estas organizaciones. Es importante resaltar que las organizaciones deben tomar en cuenta que las diversas generaciones se vienen formando en un mundo consumista, en donde la justicia organizacional convive con historias de vida llenas de frustración y organizaciones cada vez más lentas para satisfacer las demandas individuales y generacionales.

Las organizaciones tendrán que tomar en cuenta este fenómeno de frustración colectiva generada por la actual gestión del recurso humano, tratando de concatenar los diferentes factores motivacionales de las 4 generaciones y con ello reducir la incertidumbre organizacional que se ha generado actualmente en las empresas.

La principal limitación de la investigación es su generalización limitada debido al tamaño y a la amplitud de las poblaciones muestreadas (Robson, 2002). En consecuencia, la investigación es pertinente principalmente para la industria maquiladora. Además, los encuestados pudieron haber tendido a evaluar algunos valores organizacionales de manera similar con poca diferencia entre los valores de los datos, proporcionando así menos información. Sin embargo, la investigación reciente muestra que, a pesar de que las personas tienden a considerar varios valores relativamente igual en importancia y consecuentemente la evaluación de ellos de manera similar, esto no afecta a la validez general de los datos recolectados (Maio, Roese, Seligman y Katz, 1997).

Por último, el estudio muestra diferencias entre los grupos generacionales que son estadísticamente significativos. Estos resultados deben ser comparados con otros datos de las empresas maquiladoras en futuras investigaciones. Por ejemplo, las encuestas de contratación de empleados y los datos de entrevistas de salida pueden servir de base para tener una mejor idea de cómo los resultados de este estudio pueden ayudar a cada organización. También existen oportunidades para llevar a cabo investigaciones similares sobre los valores y motivaciones dentro de otras poblaciones de la fuerza laboral en México.

Ampliar la investigación puede incluir a otros países y culturas e incrementar aún más la comprensión de las generaciones, para fines tanto académicos como empresariales. Adicionalmente, esta investigación podría ser refinada mediante el uso de un método de clasificación o ipsativo que es potencialmente más predictivo de comportamiento para ilustrar mejor las preferencias individuales.

\section{Referencias}

AMAC (2014). Reporte mensual de empleos en la industria maquiladora de Ciudad Juárez. Index Juárez.

Bauman, Z. (1999). Modernidad líquida. Buenos Aires: Fondo de Cultura Económica.

Bengtson, V. L., Furlong, M. J. y Laufer, R. S. (1983). Time, aging, and the continuity of social structure: Themes and issues in generational analysis. Journal of Social Issues, 39, 45-71.

Borja, J. y Castells, M. (1997). Local y Global. La gestión de las ciudades en la era de la información. Barcelona: Taurus.

Bustillos, S., Rincones, R., Jiménez, P. y Hernández, J. (2009). Los otros nómadas: movilidad laboral internacional de personas altamente calificadas. Juárez: Universidad Autónoma de Ciudad Juárez.

CEPAL (1996). La Industria Maquiladora. Estudios e Informes de la CEPAL. México.

Cialdini, R., Petrova, P. y Goldstein, N. (2004). The hidden costs of organizational dishonesty. MIT Sloan Management Review, 45(3), 67-73.

Cooper, D. y Schindler, P. (2003). Business Research Methods (8th ed.). Boston: McGraw-Hill Irwin.

Cronbach, L. (1951). Coefficient alpha and the internal structure of tests. Psychometrika, 16(3), 297-334.

Chatman, J. (1991). Matching people and organizations: Selection and socialization in public accounting firms. Administrative Science Quarterly, 36(3), 459-484.

Dillman, D. (2000). Mail and Internet Surveys: The Tailored Design Method (2). New York: Wiley. 
Durand, J. (2005). De traidores a héroes. Políticas emigratorias en un contexto de asimetría de poder. En R. Delgado Wise y B. Knerr (Eds.), Contribuciones al análisis de la migración internacional y el desarrollo regional en México (pp. 15-38). México: Miguel Ángel Porrúa.

Earle, H. A. (2003). Building a workplace of choice: Using the work environment to attract and retain top talent. Journal of Facilities Management, 2(3), 244-257.

Eisner, S. (2005). Managing generation Y. Engineering Management Review, 39(2), 6-18.

Hackman, J. y Oldham, G. (1980). Work Redesign. Addison-Wesley: Reading, MA.

Harris, P. (2005). Boomer vs echo boomer. The work war? Training and Development, 59(5), 44-49.

Hernández, J., Gallarzo, M. y Espinoza, J. (2011). Desarrollo organizacional: enfoque latinoamericano. México: Pearson Educación.

Herzberg, F. (1959). The Motivation to Work. New York: Wiley.

Herzberg, F. (1968). One more time: How do you motivate employees? Harvard Business Review, 46(1), 53.

Juárez, A. (2007). Factores psicosociales, estrés y salud en distintas ocupaciones: Un estudio exploratorio. Investigación en Salud, 9(1), 57-64.

Karl, K. y Sutton, C. (1998). Job values in today's workforce: A comparison of public and private sector employees. Public Personnel Management, 27(4), 515-527.

Kyles, D. (2005). Managing your multigenerational workforce. Strategic Finance, 87(6), 52.

Latham, G. y Pinder, C. (2005). Work motivation theory and research at the dawn of the twenty-first century. Annual Review Psychology Journal, 56, 485-516.

Leschinsky, R. M. y Michael, J. H. (2004). Motivators and desired company values of wood products industry employees: Investigating generational differences. Forest Products Journal, 54(1), 34-39.

Lipovetsky, G. (1998). La era del vacío (10.a edición). Barcelona: Anagrama.

Lipovetsky, G. (1990). El imperio de lo efímero. Barcelona: Anagrama.

Maio, G., Roese, N., Seligman, C. y Katz, A. (1997). Rankings, ratings, and the measurement of values: Evidence for the superior validity of ratings. Basic and Applied Social Psychology, 8(2), 171-181.

Markert, J. (2004). Demographics of age: Generational and cohort confusion. Journal of Current Issues and Research in Advertising, 26(2), 11-24.

Maslow, A. (1943). A theory of human motivation. Psychological Review, 50, 370-396.

Maslow, A. (1963). The Creative Attitude. Psychosynthesis Research Foundation.

Mannheim, K. (2013). Essays Sociology Knowledge. Routledge.

McClelland, D. (1961). The Achieving Society. Princeton, NJ: Van Nostrand.

Mc Gregor, D. (1960). El lado humano de las organizaciones. México: Mcgraw-Hill.

Mckenzie, D. y Sasin, M. (2007). Migration, Remittances, Poverty and Human Capital. Conceptual and Empirical Changes. Washington: World Bank Policy Research Working Paper.

Montana, P. y Lenaghan, J. (1999). What motivates and matters most to generations X and Y. Journal of Career Planning and Employment, 59(4), 27-30.

Morton, L. (2001). Segmenting publics: Segmenting Baby Boomers. Public Relations Quarterly, 46(3), 46-47.

Morton, L. (2003). Segmenting publics: Targeting Generation X. Public Relations Quarterly, 48(4), 43-45.

Morton, L. (2004). Segmenting publics: Targeting the World War II Generation. Public Relations Quarterly, 49(1), 46-48.

O’Bannon, G. (2001). Managing our future: The Generation X factor. Public Personnel Management, 30(1), 95-110.

Peiró, J. (2004). El sistema de trabajo y sus implicaciones para la prevención de los riesgos psicosociales en el trabajo. Univ. Psychol. Bogotá, Colombia, 3(2), 179-186.

Reynolds, C. (2004). Boomers, act II. American Demographics, 26(8), 10-11.

Robbins, S. (2004). Comportamiento organizacional. México: Pearson Educación.

Robson, C. (2002). Real World Research (2nd ed.). Blackwell: Malden, MA.

Rokeach, M. (1973). The Nature of Human Values. New York: Free Press.

Santos, B. (2006). Renovar la teoría crítica y reinventar la emancipación social (encuentros en Buenos Aires). Clacso Libros.

Schlesinger, A. (1999). The Cycles of American History. Boston: Houghton Mifflin Harcourt. Copyright 1986.

Sturges, J. y Guest, D. (2004). Working to live or living to work? Work/life balance early in the career. Human Resource Management Journal, 14(4), 5-20.

Sujansky, J. (2004). Leading a multi-generational workforce. Occupational Health \& Safety, 73(4), 16-18.

Sutton, G. y Griffin, M. A. (2004). Integrating expectations, experiences, and psychological contract violations: A longitudinal study of new professionals. Journal of Occupational \& Organizational Psychology, 77, 493-514. 
Vandenberghe, C. y Peiro, J. (1999). Organizational and individual values: Their main and combined effects on work attitudes and perceptions. European Journal of Work \& Organizational Psychology, 8(4), 569-581.

Vroom, V. (1964). Work and Motivation. New York: Wiley.

Ware, J. y Grantham, C. (2003). The future of work: Changing patterns of workforce management and their impact on the workplace. Journal of Facilities Management, 2(2), 142-160.

Williams, A. y Nussbaum, J. (2001). Intergenerational communication across the life span. Mahwah, NJ: Lawrence Erlbaum Associates.

Zemke, R. (2001). Here come the millennials. Training, 38(7), 44. 